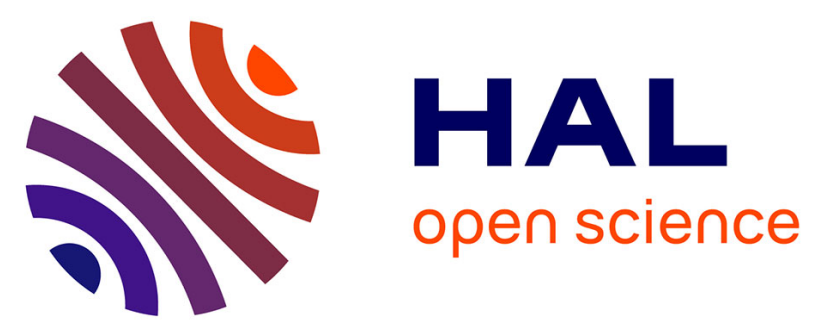

\title{
ÉVALUATION COMPLÈTE DE L'ACOUSTIQUE D'UNE SALLE: CAS DU THÉÂTRE MUNICIPAL DU MANS
}

X. Meynial, J. Polack, G. Dodd

\section{- To cite this version:}

X. Meynial, J. Polack, G. Dodd. ÉVALUATION COMPLÈTE DE L'ACOUSTIQUE D'UNE SALLE: CAS DU THÉÂTRE MUNICIPAL DU MANS. Journal de Physique IV Proceedings, 1992, 02 (C1), pp.C1-155-C1-158. 10.1051/jp4:1992130 . jpa-00251201

\section{HAL Id: jpa-00251201 https://hal.science/jpa-00251201}

Submitted on 1 Jan 1992

HAL is a multi-disciplinary open access archive for the deposit and dissemination of scientific research documents, whether they are published or not. The documents may come from teaching and research institutions in France or abroad, or from public or private research centers.
L'archive ouverte pluridisciplinaire HAL, est destinée au dépôt et à la diffusion de documents scientifiques de niveau recherche, publiés ou non, émanant des établissements d'enseignement et de recherche français ou étrangers, des laboratoires publics ou privés. 


\title{
EVALUATION COMPLETE DE L'ACOUSTIQUE d'UNE SALLE : CAS DU THÊtTRE MUNICIPAL DU MANS
}

\author{
X. MEYNIAL, J.D. POLACK et G. DODD* \\ Laboratoire d'Acoustique, Université du Maine, BP. 535, Avenue O. Messiaen, F-72017 Le Mans \\ cedex, France \\ "Acoustics Research Centre, School of Architecture, University of Auckland, Private Bag, Auckland, \\ New Zealand
}

\begin{abstract}
First we present the equipment used for objective measurements both in the hall and in the 1:50 model, the accuracy of the measurements, and the results of the measurements. Examination of these results lead us to distinguish between two possible uses of models in room scoustics corresponding to two levels of precision in model realisation.

Results of the subjective tests carried out in the theatre are looked at in terms of their correlations with objective measurements, and observations on the relevance of such tests for characterising a hall are derived. Specially, we believe the audition-vision interaction can't be ignored.
\end{abstract}

\begin{abstract}
Nous exposons dans un premier temps les dispositifs utilisés pour les mesures objectives dans la salle et dans la maquette au 1:50ème, la précision des mesures effectuées, puis présentons les résultats. L'examen de ceux-ci nous amène à distinguer deux utilisations possibles des maquettes en acoustiques des salles correspondant à deux niveaux de précision dans la réalisation des maquettes.

L'analyse des résultats des tests subjectifs menés au Théâtre induisent quelques observations sur leur corrélations avec les mesures objectives, et sur la pertinence de tels tests pour la caractérisation d'une salle. En particulier, l'intéraction audition-vision nous paraît devoir être prise en compte.
\end{abstract}

\section{INTRODUCTION}

Le Laboratoire d'Acoustique de l'Université du Maine a été sollicité en 1989 pour la rénovation du Théâtre Municipal du Mans (1009 places). L'étude acoustique fût conduite en partie sur une maquette au 1:50ème du théâtre. Les mesures acoustique dans la salle et dans la maquette ont été faites avec le système MIDAS [1,2] qui permet l'utilisation de maquettes en air ambiant grâce à une compensation numérique de l'atténuation de propagation. Nous présentons ici certaines des observations auxquelles ce travail nous a amene, tant sur le plan des mesures objectives sur maquette et à pleine échelle, que sur cehui de la caractérisation subjective d'une salle.

\section{MESURES OBJECTIVES. DISPOSITIF ET PRECISION DES MESURES}

Les mesures sont basées sur des réponses impulsionnelles de la salle. Dans la maquette, des impulsions sonores brèves (environ 20 micro-sec) sont générées à l'aide d'un éclateur, et captées par un microphone électrostatique $1 / 8^{\prime \prime}$. La bande de fréquence utile de cette chaîne est environ 5$150 \mathrm{kHz}$; ce qui correspond à $100 \mathrm{~Hz}-3 \mathrm{kHz}$ à pleine échelle. Les indices sont donc évalués dans les octaves $125 \mathrm{~Hz}$ à $2 \mathrm{kHz}$ équivalent pleine échelle.

A pleine échelle, un pistolet d'alarme génère les impulsions sonores qui sont captées par un 
microphone électret. Notons que l'utilisation de sources impulsives à été préférée à l'emploi de sources entretenues (alimentées par un bruit pseudo-aléatoire combiné à la déconvolution de Hadamard) car:

* il n'existe pas de source entretenue pour les mesures au 1:50ème

* la directivité des sources impulsives est mieux maitrisée

* les échogrammes obtenus avec les sources impulsives sont beaucoup plus lisibles car le support des impulsions est beaucoup plus court

La répétabilité de ce type de source est bonne en terme d'allure spectrale, moins bonne quand aux niveaux sonores générés $( \pm l \mathrm{lB})$.

MIDAS dispose d'un système de compensation des mesures par l'ènergie du spectre de la chaine de mesure (par rapport à une mesure de référence du son direct). La mise en service de cette compensation peut affecter très sensiblement les valeurs des indices. Afin de minimiser l'influence des transducteurs dans les comparaisons maquette/peine échelle, cette compensation a été utilisée pour toutes les mesures.

Nous avons utilisé les indices monoraux couramment cités dans la littérature : EDT, RT, C80, Tc, RASTI, AMPLI (G à une constante additive près). Il est clair que cette liste est très redondante [3]. Nous avons évalué les incertitudes des mesures des indices effectuées dans la maquette dues à plusieurs causes :

a. la répétabilité (sans replacement des transducteurs): écarts-types observés

b. la directivité du microphone : écarts maximaux des indices mesurés dans la maquette avec utilisation d'un cône au lieu de la grille du micro (1/8")

c. placement du microphone : écarts-types des indices pour une exploration de $1 \mathrm{~cm} 3$ dans la maquette

d. réglage des paramètres logiciels : écarts-types observés

Les déviations obtenues sont résumées dans le tableau 1, la dernière ligne étant une estimation de la précision générale des mesures. Cette estimation est proche de celles obtenues à pleine échelle par différents auteurs.

Il faut toutefois noter que le placement du microphone est particulièrement sensible lorsque l'on se trouve dans des géométries particulières. Par exemple, la source étant située au centre de la scène, un léger déplacement du micro de part et d'autre de l'axe central de la salle provoque de grandes variations des indices dues au fait que les deux réflexions issues des murs latéraux sont distinctes (énergie 2E), ou s'additionnent (énergie 4E), ou s'annulent partiellement (énergic E). On touche là à une faiblesse inhérente aux indices monoraux qui ne rendent pas compte de l'écoute binaurale et des effets de diffraction de la tête et du buste de l'auditeur.

\begin{tabular}{|c|c|c|c|c|c|c|c|}
\hline \multicolumn{3}{|c|}{$\triangle \mathrm{EDT}$} & \multirow{2}{*}{$\begin{array}{c}\Delta \mathrm{RT} 30 \\
1 \% \\
1 \% \\
\end{array}$} & \multirow{2}{*}{$\begin{array}{l}\Delta \mathrm{C} 80 \\
0,1 \mathrm{~dB} \\
0,2 \mathrm{~dB}\end{array}$} & \multirow{2}{*}{\begin{tabular}{r|}
$\Delta \mathrm{Tc}$ \\
$2 \%$ \\
$4 \%$ \\
\end{tabular}} & \multirow{2}{*}{$\begin{array}{r}\Delta \text { Ampli } \\
0,1 \mathrm{~dB} \\
0,2 \mathrm{~dB}\end{array}$} & \multirow{2}{*}{$\begin{array}{c}\Delta \text { RASTI } \\
0 \% \\
\end{array}$} \\
\hline $\begin{array}{l}\text { a. Répétabilité } \\
\text { sans replacement }\end{array}$ & $\begin{array}{r}\text { (pleine bande) } \\
\text { (octaves) }\end{array}$ & $\begin{array}{l}1 \% \\
1 \%\end{array}$ & & & & & \\
\hline $\begin{array}{l}\text { b. Directivité } \\
\text { microphone }\end{array}$ & $\begin{array}{l}\text { (pleine bande) } \\
\text { (octave 2kHz) }\end{array}$ & $\begin{array}{l}3 \% \\
6 \%\end{array}$ & $\begin{array}{l}2 \% \\
3 \%\end{array}$ & $\begin{array}{l}1,3 \mathrm{~dB} \\
1,9 \mathrm{~dB}\end{array}$ & $\begin{array}{l}10 \% \\
14 \%\end{array}$ & $\begin{array}{l}0,7 \mathrm{~dB} \\
1,2 \mathrm{~dB}\end{array}$ & $\begin{array}{l}0 \% \\
\cdots--\end{array}$ \\
\hline $\begin{array}{l}\text { c. Placement } \\
\text { microphone }\end{array}$ & $\begin{array}{l}\text { (pleine bande) } \\
\text { (octave 125Hz) }\end{array}$ & $\begin{array}{r}6 \% \\
12 \%\end{array}$ & $\begin{array}{l}4 \% \\
5 \%\end{array}$ & $\begin{array}{l}0,5 \mathrm{~dB} \\
1,1 \mathrm{~dB}\end{array}$ & $\begin{array}{l}6 \% \\
10 \%\end{array}$ & $\begin{array}{l}0,5 \mathrm{~dB} \\
0,9 \mathrm{~dB}\end{array}$ & $\begin{array}{l}1 \% \\
\cdots--\end{array}$ \\
\hline $\begin{array}{l}\text { d. Paramètres } \\
\text { logiciels }\end{array}$ & $\begin{array}{c}\text { (pleine bande) } \\
\text { (octaves) }\end{array}$ & $\begin{array}{l}2 \% \\
7 \%\end{array}$ & $\begin{array}{l}2 \% \\
4 \%\end{array}$ & $\begin{array}{l}0,2 \mathrm{~dB} \\
0,8 \mathrm{~dB}\end{array}$ & $\begin{array}{l}2 \% \\
5 \%\end{array}$ & $\begin{array}{l}0,1 \mathrm{~dB} \\
0,5 \mathrm{~dB}\end{array}$ & $\begin{array}{l}1 \% \\
\cdots-\end{array}$ \\
\hline $\begin{array}{l}\text { Précision } \\
\text { générale (mo) }\end{array}$ & $\begin{array}{r}\text { (pleine bande) } \\
\text { nne des octaves) }\end{array}$ & $\begin{array}{l}7 \% \\
10 \%\end{array}$ & $\begin{array}{l}5 \% \\
7 \%\end{array}$ & $\begin{array}{l}0,7 \mathrm{~dB} \\
1,4 \mathrm{~dB}\end{array}$ & $\begin{array}{l}7 \% \\
10 \%\end{array}$ & $\begin{array}{l}0,6 \mathrm{~dB} \\
1,2 \mathrm{~dB}\end{array}$ & $1 \%$ \\
\hline
\end{tabular}

Tableau 1: Effet des principales incertitudes de mesure sur les indices objectifs et précision générale résultante 


\section{RESULTATS DES MESURES OBJECTIVES}

Les allures des échogrammes observées dans la maquette sont en général très proche de celles observées à pleine échelle. Toutefois, de fortes différences sont observées dès que la surface n'est pas correctement reproduite dans la maquette, $œ$ qui est le cas au 2 ème balcon.

L'écart entre les TR moyens mesurés dans la salle et dans la maquette est de $2 \%$ dans les octaves $125 \mathrm{~Hz}$ et $250 \mathrm{~Hz}$, puis crôt pour atteindre $10 \%$ à $500 \mathrm{~Hz}$ et $20 \%$ à $1 \mathrm{kHz}$ et $2 \mathrm{kHz}$. Cet excès d'absorption aux hautes fréquences dans la maquette est dû en partie à l'absorption viscothermique aux parois $(\approx 5 \%$ à $100 \mathrm{kHz})$, mais surtout à une mauvaise modélisation du rideau de fond de scène qui n'avait fait l'objet d'aucune étude préalable (le TR à $2 \mathrm{kHz}$ augmente de $20 \%$ lorsqu'on enlève le rideau). Ceci met en évidence la nécessité d'une modélisation fine de l'absorption pour une mesure quantitative des indices.

En conséquence des observations ci-dessus, nous analysons les indices mesurés en termes de classement par siège. Ainsi, dans les résultats (pleine bande) présentés par le tableau 2, nous avons distingué 4 groupes, suivant l'accord entre maquette et salle pleine échelle.

Tableau 2: Résultats des mesures des indices objectifs pleine-bande au théâtre et dans la maquette. Accord entre les deux types de mesure:

*.* Accord sur une valeur plutôt forte de l'indice

- Accord sur une valeur moyenne de l'indice

- Accord sur une valeur plutôt faible de l'indice

$\mathrm{xxxx}$ Désaccord sur la valeur de l'indice

\begin{tabular}{|c|c|c|c|c|c|c|c|c|}
\hline \multirow{2}{*}{\multicolumn{9}{|c|}{ 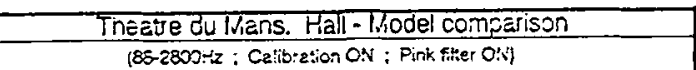 }} \\
\hline \multicolumn{4}{|c|}{ (86-2800:1z : Catib:ztion ON ; Pink fiter ON) } & & & & & \\
\hline & & \multicolumn{3}{|c|}{ Stails } & \multicolumn{2}{|c|}{ ist balcony } & \multicolumn{2}{|c|}{$2 n j$ talociny } \\
\hline & & $\overline{E 1}$ & L1 & $\bar{K} 24$ & Q1 & UE4 & $\overline{\mathrm{BB1}}$ & $\overline{K K 22}$ \\
\hline $\begin{array}{l}\text { EDT } \\
\text { (s) }\end{array}$ & $\begin{array}{l}\text { hall } \\
\text { model }\end{array}$ & $\begin{array}{l}1,05 \\
0,92\end{array}$ & $\begin{array}{l}0,98 \\
0,84 \\
0 . .\end{array}$ & $\begin{array}{l}0,88 \\
0,80\end{array}$ & $\begin{array}{l}0,77 \\
0,75\end{array}$ & $\begin{array}{l}0,67 \\
0,53\end{array}$ & $\begin{array}{l}0,97 \\
0,87 \\
\therefore . .\end{array}$ & $\begin{array}{l}0,80 \\
0,71\end{array}$ \\
\hline $\begin{array}{l}\text { PT } \\
\text { (s) }\end{array}$ & $\begin{array}{c}\text { hall } \\
\text { model }\end{array}$ & $\begin{array}{l}1,19 \\
1,00\end{array}$ & $\begin{array}{l}1,22 \\
1,10\end{array}$ & $\begin{array}{l}1,25 \\
1,08\end{array}$ & $\begin{array}{l}1,24 \\
0,98 \\
\end{array}$ & $\begin{array}{l}1,10 \\
1,13\end{array}$ & $\begin{array}{l}1,10 \\
1,16 \\
\end{array}$ & $\begin{array}{l}1,07 \\
1,00\end{array}$ \\
\hline EDT/RT & mall & $\begin{array}{l}0,88 \\
0,92 \\
\ldots .\end{array}$ & $\begin{array}{l}0,80 \\
0,76 \\
: .\end{array}$ & $\begin{array}{l}0,70 \\
0,74\end{array}$ & $\begin{array}{l}0,62 \\
0,77 \\
\end{array}$ & $\begin{array}{l}0,61 \\
0,47\end{array}$ & $\begin{array}{l}0,88 \\
0,75 \\
x \times x=x\end{array}$ & $\begin{array}{l}0,75 \\
0,71 \\
: .\end{array}$ \\
\hline $\begin{array}{l}\mathrm{C} 80 \\
\text { (dB) }\end{array}$ & $\begin{array}{c}\text { hall } \\
\text { model }\end{array}$ & $\begin{array}{l}3,2 \\
4,4\end{array}$ & $\begin{array}{r}4,4 \\
5,8 \\
x \text { xexux }\end{array}$ & $\begin{array}{l}5,9 \\
5,6 \\
\therefore\end{array}$ & $\begin{array}{l}6,1 \\
5,6 \\
\therefore:\end{array}$ & $\begin{array}{l}6,6 \\
8,1 \\
.\end{array}$ & $\begin{array}{l}1,3 \\
2,8\end{array}$ & $\begin{array}{c}5,3 \\
5,6 \\
-5\end{array}$ \\
\hline $\begin{array}{l}T c \\
\text { (ms) }\end{array}$ & $\begin{array}{l}\text { hall } \\
\text { model }\end{array}$ & $\begin{array}{r}73 \\
64 \\
\therefore .\end{array}$ & $\begin{array}{l}58 \\
53 \\
.\end{array}$ & 51 & $\begin{array}{l}56 \\
52 \\
?\end{array}$ & $\begin{array}{l}44 \\
40\end{array}$ & $\begin{array}{r}78 \\
69 \\
\cdots\end{array}$ & $\begin{array}{l}54 \\
55\end{array}$ \\
\hline $\begin{array}{l}\text { Ampli } \\
\text { (dB) }\end{array}$ & $\begin{array}{l}\text { hall } \\
\text { model }\end{array}$ & $\begin{array}{l}8,3 \\
6,8 \\
. .\end{array}$ & $\begin{array}{l}3,2 \\
4,0 \\
? .\end{array}$ & $\begin{array}{l}3.5 \\
2.9 \\
.9\end{array}$ & \begin{tabular}{l}
7,5 \\
5,0 \\
\hdashline..
\end{tabular} & $\begin{array}{l}6,2 \\
4,4 \\
-\end{array}$ & $\begin{array}{l}3,9 \\
3,2 \\
-\end{array}$ & $\begin{array}{r}3,8 \\
-0,6\end{array}$ \\
\hline STI & $\begin{array}{l}\text { hall } \\
\text { model }\end{array}$ & $\begin{array}{l}0,58 \\
0,60\end{array}$ & $\begin{array}{l}0,63 \\
0,66\end{array}$ & $\begin{array}{l}0,65 \\
0,64 \\
\end{array}$ & $\begin{array}{l}0,63 \\
0,66\end{array}$ & $\begin{array}{l}0,68 \\
0,69 \\
\end{array}$ & $\begin{array}{l}0,57 \\
0,59 \\
\end{array}$ & $\begin{array}{l}0,65 \\
0,63 \\
\end{array}$ \\
\hline
\end{tabular}

Ces résultats, nous amènent à faire quelques observations :

* La différence sur la réverbération a déjà été discutée

* La plupart des désaccord correspondent à des places situées sur l'axe de symétrie de la salle. Le choix des places de test n'était donc pas judicieux.

* Des désaccords apparaissent au 2 ème balcon, ce qui est dû à une mauvaise modélisation géométrique du plafond.

* Les tendances sont en général bien respectées, surtout pour AMPLI et STI

- Les écarts types des indices mesurés aux 7 places de test sont similaires dans la salle et à pleine échelle.

En conclusion de ces mesures objectives, nous dirons que l'emploi de maquettes simples (et bon marché) tout en étant assez précises sur le plan géométrique donne de bons résultats quant aux échogrammes et aux tendances sur les indices. De telles maquettes sont utilisables aux stades premiers de la conception d'une salle pour la mise au point de formes, et dans un cadre éducatif pour la mise en évidence de phénomènes acoustiques. Dans ce dernier cas, des maquettes extrêmement simples peuvent être utilisées, éventuellement réalisées par les étudiants eux-mêmes.

Pour une caractérisation quantitative précise, des maquettes plus évoluées (donc plus chères) doivent être réalisées -éventuellement à échelle plus grande que 1:50-reproduisant fiđèlement l'absorption acoustique dans la salle. Dans ce but, il serait souhaitable d'élargir nos connaissances sur l'absorption acoustique des matériaux utitisés dans la construction des maquettes. 


\section{COMPARAISON DES MESURES OBJECTIVES AVEC LES TESTS SUBJECTIFS}

L'évaluation subjective du Théâtre a été conduite avec 30 sujets non-expérimentés et deux ensembles musicaux, autour des 7 sièges retenus pour les mesures objectives. Les questionnaire a été adapté de celui de Barron [4].

Il ressort de l'analyse des résultats que l'Impression Générale est fortement corrélée avec tous les attributs perceptifs "orientés", c'est à dire ceux pour lesquels l'un des extrêmes de l'échelle a une connotation positive. Plus particulièrement, les bonnes places sont celles où la sonie est élevée. Effectivement, l'analyse détaillée des résultats objectifs montre que la classification des places selon l'Impression Générale est la même que celle du niveau sonore (AMPLI), surtout si l'on ne retient que le niveau sonore aux basses fréquences $(125 \mathrm{~Hz}$ et $250 \mathrm{~Hz})$. Cette dernière est fortement liée à la distance à la source et à l'impression visuelle, car c'est aux places où l'on "domine" la scène que le son direct ne subit aucun masquage.

Ce type d'analyse réduit à une seule salle (et non à une comparaison de salles entre elles) a aussi montré la difficulté de mettre au point un questionnaire allant au delà de l'Impression Générale, et la difficulté d'obtenir des corrélations "robustes". C'est ainsi que le petit nombre de places (7) étudiées au Théâtre réduit à six le nombre d'attributs perceptifs analysables simultanément. Dans ces conditions, les corrélations entre attributs perceptifs ne sont pas étonnantes, ni le fait que seul le niveau sonore émerge comme indice.

Enfin, la plupart des techniques d'analyse évacuent la variabilité des réponses des auditeurs, ce qui fait conclure à une grande homogénéité de la salle.

\section{CONCLUSION ET PERSPECTIVES}

Le cas du Théâtre du Mans nous a convaincu de l'intérêt et de la pertinence de l'utilisation de maquettes et du système MIDAS en acoustique architecturale. Il démontre la nécessité d'une modélisation fine de la géométrie et de l'absorption pour avoir des mesures quantitativement pertinentes dans les maquettes. Un outil permettant à l'expérimentateur de maîtriser l'absorption dans la maquette doit être développé.

Contrairement à la tendance actuelle, nous avons utilisé des sources sonores impulsives. Leur brièveté assure une bonne lecture des échogrammes et minimise les problèmes de directivité. Dans les maquettes encore plus qu'à pleine échelle, les transducteurs imposent des limitations (en fréquence, directivité,...) qu'il conviendrait de repousser.

Par ailleurs, cette étude souligne la difficulté de la caractérisation subjective d'une salle. L'homogénéité du Théâtre en est certes responsable, mais l'influence d'une interaction visionaudition ne peut être écartée. Ceci plaide en faveur d'une simulation acoustique et visuelle des salles, direction que nous souhaitons poursuivre.

[1] J.D.POLACK, A.H.MARSHALL, G.DODD, Digital evaluation of the acoustics of small models : The MIDAS package, J.Acoust. Soc. Am. 85 (1989), 185-193

[2] X.MEYNIAL, J.D.POLACK, G.DODD, A.H.MARSHALL, All-scale model measurements : The MIDAS system, 121 st ASA Conference, Baltimore, April 1991.

[3] L.CREMER, H.A.MÚLLER, T.J.SHULTZ, Principles and applications of room acoustics, (Applied Science, 1982)

[4] M.BARRON, Subjective study of British symphony concert halls, Acustica 66 (1988), $1-14$ 